\title{
Un épisode de l'histoire de la couleur au cinéma : le procédé Keller-Dorian et les films lenticulaires
}

An episode in the history of film colour : the Keller-Dorian process and lenticular films

\section{François Ede}

\section{(2) OpenEdition}

\section{Journals}

Édition électronique

URL : https://journals.openedition.org/1895/4787

DOI : $10.4000 / 1895.4787$

ISSN : 1960-6176

Éditeur

Association française de recherche sur l'histoire du cinéma (AFRHC)

Édition imprimée

Date de publication : 1 décembre 2013

Pagination : 187-202

ISBN : 978-2-37029-071-7

ISSN : 0769-0959

Référence électronique

François Ede, «Un épisode de l'histoire de la couleur au cinéma : le procédé Keller-Dorian et les films lenticulaires », 1895. Mille huit cent quatre-vingt-quinze [En ligne], 71 | 2013, mis en ligne le 01 décembre 2016, consulté le 17 avril 2022. URL : http://journals.openedition.org/1895/4787 ; DOI : https:// doi.org/10.4000/1895.4787 


\section{Un épisode de l'histoire de la couleur au cinéma : le procédé Keller-Dorian et les films lenticulaires}

François Ede

Il y a une vingtaine d'années, j'ai eu l'occasion de recueillir le témoignage de deux anciens ingénieurs chimistes, aujourd'hui disparus: René Pommier et Georges Bonnerot. Tous deux diplômés de l'Institut de Chimie de Paris en 1922, ils firent leur carrière dans l'industrie cinématographique. À ce titre, ils participèrent aux recherches sur la couleur, notamment sur le procédé Keller-Dorian. Ces informations inédites et la consultation de leurs archives, qu'ils me confièrent et que j'ai, à mon tour, déposées à la Cinémathèque française en 2006, ont constitué une source précieuse et ont largement contribué à lever un coin du voile sur un épisode relativement peu connu de l'histoire du cinéma.

Les recherches de Lippmann et le procédé Keller-Dorian-Berthon

Les recherches du physicien Gabriel Lippmann sur la reproduction spectrale des couleurs par la méthode interférentielle lui valent un prix Nobel de physique en 1908 pour sa découverte des propriétés ondulatoires de la lumière. Cette même année 1908, Lippmann, dans une note à l'Académie des Sciences intitulée: Épreuves réversibles. Photographies intégrales ${ }^{1}$, définit par une recherche originale, un nouveau moyen d'obtenir "toute cette variété qu'offre la vue directe des objets».

Le mode opératoire qu'il décrit est très clair. Il consiste à combiner une émulsion photosensible avec un support recouvert d'un réseau de multiples lentilles convergentes de très petites dimensions. Pour ce faire, il suggère, avant de coucher l'émulsion de presser le cellulö̈ à chaud avec "une sorte de machine à gaufrer", ou encore de coller sur le support des "sphérules de verre qui forment lentilles». Ce réseau de minuscules objectifs permet d'enregistrer une multitude d'images de très petite taille, sans chambre noire. L'image, une fois exposée, développée et dont on a tiré un positif, peut être observée en vertu de la loi du retour inverse de la lumière, c'est pourquoi il parle "d'épreuves réversibles":

Supposons l'œil placé du côté antérieur [celui des microlentilles], et l'épreuve placée par transparence en lumière diffuse, [...] l'œil verra alors à la place du système des petites images une seule image résultante projetée dans l'espace en vraie grandeur. [...] De plus, leur aspect change avec la position du spectateur, comme si celui-ci se trouvait en présence de la réalité.

1. Gabriel Lippmann, Comptes rendus des séances de l'Académie des Sciences, $1^{\mathrm{er}}$ semestre1908, t. CXLVI, $\mathrm{n}^{\circ}$ 9, p. 446-451. Bibliothèque de l'Institut, Paris. Sauf mention contraire, les citations qui suivent proviennent de la même source. 
Lippmann ouvrait ainsi une nouvelle voie permettant d'enregistrer des images dites "auto-stéréoscopiques». Eugène Estanave, en 1925 et, plus tard, Maurice Bonnet en 1939 produiront de magnifiques images en «relief changeant» [Fig. 1].

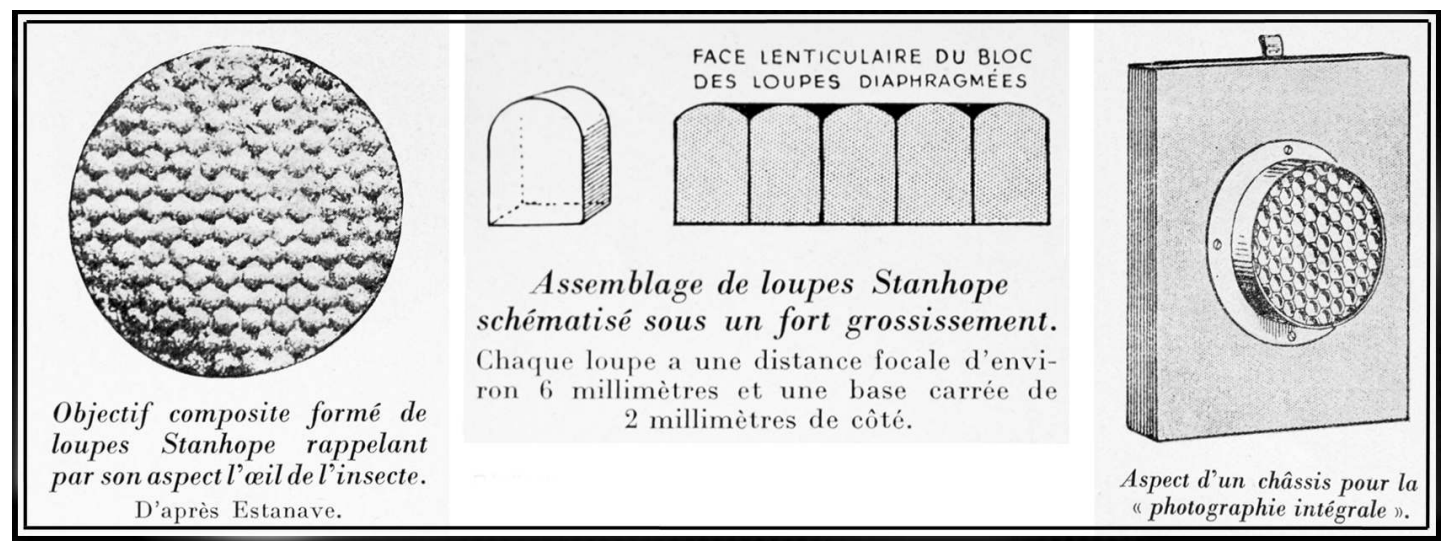

Fig. 1 : Procédé Estanave, circa1925.

Si Lippmann ne fait aucune allusion à la possibilité d'enregistrer ces images en couleurs, cette alternative est implicitement contenue dans sa note. Rodolphe Berthon est un ingénieur et astronome lyonnais. Il reprend l'idée cardinale de Lippmann et l'adapte non pas à la photographie en relief, mais à la cinématographie des couleurs.

Le réseau de micro-lentilles, est, cette fois, associé à un objectif primaire de prise de vue dans lequel est placé un filtre à bandes rouges, vertes et bleues (RVB), au point d'émergence de la pupille de l'objectif. La focale de chacun de ces micro-objectifs est calculée pour former l'image du filtre, de sorte que chaque petite lentille recueille l'image virtuelle du sélecteur trichrome, permettant le codage des couleurs. On peut ici parler d'un codage analogique, car en l'absence de filtre, on obtient une image en noir et blanc. Chaque photogramme est enregistré, non pas directement sur l'émulsion, mais à travers le support gaufré d'une pellicule inversible panchromatisée ${ }^{2}$.

La restitution des couleurs en projection est obtenue là encore, selon le principe du retour inverse de la lumière [Fig. 2].

Berthon dépose de nombreux brevets en France, au Royaume-Uni et aux États-Unis ${ }^{3}$. Le brevet

2. Un film inversible est un original positif obtenu au développement par réexposition à la lumière. Les émulsions ordinaires n'étant sensibles qu'au bleu et à l'ultra-violet, la panchromatisation permettait de les sensibiliser à l'ensemble des radiations du spectre au moyen de colorants appropriés incorporés dans l'émulsion.

3. Berthon envisagea des solutions alternatives avec Maurice Audibert, dont un procédé comportant trois images de sélection trichrome sur une pellicule de $65 \mathrm{~mm}$ de largeur. Quelques essais sont conservés à la Cinémathèque française. 


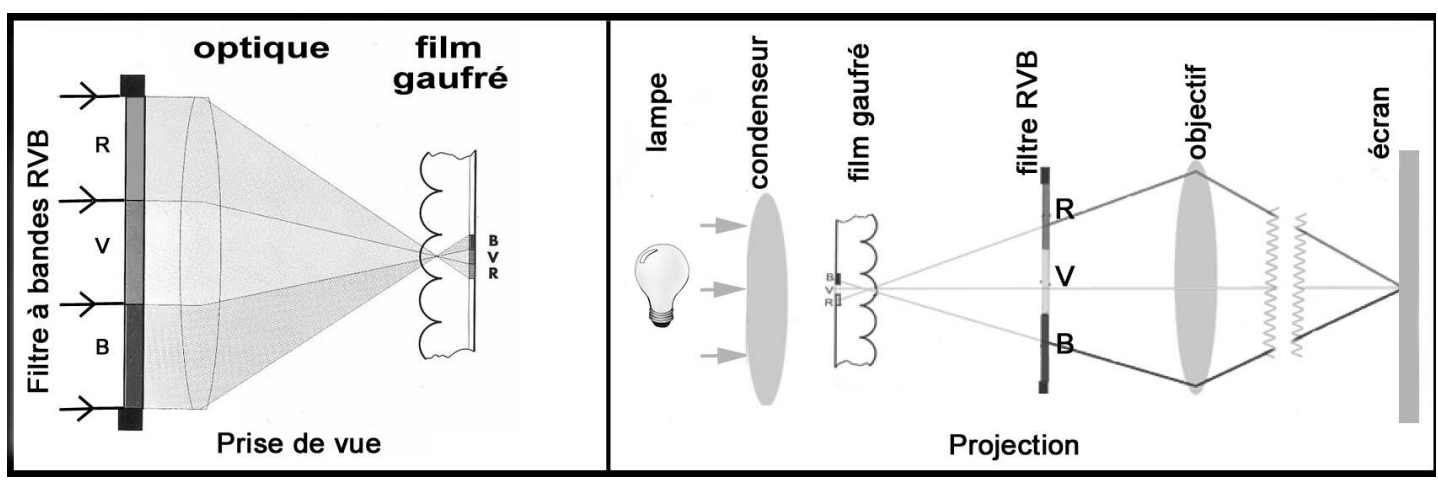

Fig. 2: Schéma de principe du procédé lenticulaire, prise de vues et projection.

français initial date du $1^{\text {er }}$ mai $1908^{4}$. Près de quinze ans vont pourtant s'écouler entre sa publication et les premières expérimentations attestées qui eurent lieu au début des années 1920. On peut avancer quelques hypothèses expliquant cette longue période de gestation, à commencer par les difficultés rencontrées pour la mise au point d'une machine de gaufrage permettant une finesse de gravure jamais atteinte. Les différents brevets indiquent que les dioptres mesurent 40 micromètres, soit 25 dioptres/ $\mathrm{mm}$ pour permettre la fusion rétinienne. À la recherche d'une solution pratique, Berthon s'adresse à l'industriel Albert Keller-Dorian. Ce dernier a pris la succession de son père Charles Keller et dirige une importante entreprise de gravure et d'impression sur tissu et papier à Mulhouse. Albert KellerDorian, féru de techniques nouvelles, a modernisé l'entreprise familiale et y a développé la conception et la fabrication de machines de gravure performantes. Sa curiosité pour les procédés photographiques l'amène à fréquenter les sociétés scientifiques. L'industriel fait de fréquents séjours à Lyon où il a ouvert une succursale en 1888. Keller-Dorian s'intéresse à l'invention de Berthon et va étudier les solutions pour mettre au point une machine de gaufrage qui constitue la clé de voûte du procédé lenticulaire.

Les premières tentatives de gravure sur film eurent lieu avant 1914, mais ne sont pas documentées. L'historien, cinéaste et critique Jean Mitry donne quelques précisions techniques dans son Histoire du cinéma:

À l'origine, les éléments du gaufrage avaient une forme hexagonale d'un diamètre de 50 . En 1913, on utilisa une matrice gravée en creux imprimant dans la matière même du support des éléments

4. Le brevet Berthon d'origine : Perfectionnements aux procédés de photographie trichrome » no 399762, déposé le $1^{\text {er }}$ mai 1908, est publié le $1^{\text {er }}$ juillet 1909 (INPI). Les brevets anglais et américains datent également de 1909: brevet anglais $\mathrm{n}^{\circ}$ 10611: "Origin of Lenticular Process" [origine du procédé lenticulaire] et brevet nord-américain : US 992151 A: "Apparatus for color Photography" [appareil pour la photographie en couleurs]. Réf: www.google. com.mx/search ?tbm=pts\&hl=fr\&q=416\%2C161\#hl=fr\&q=rodolphe+berthon\&tbm=pts [date de la dernière consultation : octobre 2013]. 
lenticulaires de forme cylindrique à raison de 1500 au millimètre carré. Là encore, l'établissement de cette matrice de platine, beaucoup plus fine que la précédente, demanda des années de travail 5 .

Le premier conflit mondial va interrompre ces premières recherches. À l'issue de la guerre, la société Keller-Dorian, comme beaucoup d'entreprises industrielles, est économiquement affaiblie. Or, la mise au point d'un procédé de film en couleurs représente d'importants investissements. S’il continue d'apporter son concours à Berthon pour la continuation des travaux de recherche, Keller-Dorian n'est plus en mesure de porter seul le développement de l'invention à l'échelle industrielle. En 1921, il est âgé de 75 ans et bien qu'il continue d'assurer la direction de son entreprise, il veut à la fois préparer sa succession et poursuivre la modernisation de la société. Pour augmenter le capital de son entreprise, il en modifie les statuts et la transforme en société anonyme par actions. Désormais, tout projet impliquant une mise de fonds conséquente sera soumis à l'approbation des actionnaires représentés par un conseil d'administration.

Il faut attendre 1922 pour que quelques courtes bandes d'essais soient présentées par Berthon à un cercle restreint. C'est à Mulhouse, au début de l'année 1923, qu'a lieu la première projection publique. L'hebdomadaire corporatif les Spectacles, destiné aux exploitants, relate succinctement l'événement :

Il y a un peu plus d'un an, le public de Mulhouse était convié à assister, dans une des salles de conférence de la ville, à une démonstration de cinématographie en couleurs, présentée par son inventeur M. Albert Keller-Dorian, cette invention est appelée à se développer, car elle tient compte des conditions de simplicité, de commodité nécessaires à sa diffusion ${ }^{6}$.

Un encart paru avec l'article annonce officiellement la création «de la Société Anonyme de films en couleurs Keller-Dorian " ${ }^{7}$. Par un hasard malheureux, la création de la société va coïncider avec le décès d'Albert Keller-Dorian, en juillet 1924. Le siège social de La Société du Film en Couleurs est d'abord établi à Paris, dans des locaux de la société Optis, (fabricant d'optique), au 25, rue Saint-Fargeau (Paris, $\mathrm{xx}^{\mathrm{e}}$ ). Cette domiciliation n'est pas fortuite car Berthon avait travaillé sur la conception d'optiques pour cette société. À partir de 1925, les bureaux de la Société seront transférés au 42, rue d'Enghien (Paris, $\mathrm{x}^{\mathrm{e}}$ ).

Une équipe de recherche a été constituée. Elle est composée d'un directeur technique (Abel-Pierre Richard), de deux personnalités connues: le professeur Henri Chrétien ingénieur en optique et Léopold Löbel, ingénieur chimiste, assisté de Georges Bonnerot et de René Pommier. La fabrication

5. Jean Mitry, Histoire du cinéma, t. 2: le Cinéma pendant la guerre (1915-1919), Paris, Éditions universitaires, 1967, pp. 80-82.

6. Georges Bergner, "Une contribution de l'Alsace à la science cinématographique française", les Spectacles, $\mathrm{n}^{\circ} 35$, 29 août 1924, p. 13.

7. Environ 6 millions d'Euros (source INSEE, indicateur de pouvoir d'achat de l'Euro et du franc). 
des molettes de gaufrage est alors assurée par Alphonse Gary. Enfin, un ingénieur en optique, Isaac Kitroser, assiste le professeur Chrétien.

Assez curieusement, Berthon n'est pas intégré à cette équipe, bien qu'il continue de participer occasionnellement aux travaux. Seules quelques informations parues dans la presse corporative sur la constitution de la société nous renseignent sur la nature de ses rémunérations : «[...] Le capital [est] de 7 millions en actions de 500 francs, dont 4000 attribuées aux apporteurs qui reçoivent en outre 1.500 .000 fr. en espèces et 2000 parts bénéficiaires " ${ }^{8}$. En bon industriel, Keller-Dorian a su valoriser le coût des recherches antérieures menées dans son entreprise. Cette prime aux «apporteurs» sera par la suite jugée très excessive par les actionnaires, car beaucoup d'entre eux, attirés par ce qu'ils croyaient être un nouvel Eldorado, ne virent pas tout de suite que le procédé était encore loin de pouvoir être exploité par l'industrie. Il faut noter que la presse de l'époque a contribué largement à répandre le mythe d'une invention dont l'avenir était immédiatement assuré. Ces effets d'annonce ont attiré de nombreux boursicoteurs alléchés par la perspective de revenus mirobolants. Ainsi, le Journal des Finances indique que "la Société Keller-Dorian peut évidemment escompter des bénéfices considérables " ${ }^{9}$. Les actionnaires, sans doute ignorants des difficultés techniques réelles, accueillirent mal par la suite des bilans financiers en déficit chronique.

Dans un article paru sept ans après la création de la Société du Film en Couleurs, Abel Pierre Richard, directeur technique de la Société, dresse un constat sans complaisance et fait part des nombreuses difficultés qu'il fallut surmonter avant de parvenir à des résultats :

Les premiers essais furent réalisés rue du Chemin Vert. Le laboratoire se trouvait dans un grenier où était montée la première machine à gaufrer, fabriquée par Albert Keller-Dorian. Cette machine avait un vice rédhibitoire, elle ne permettait de faire que de très courts métrages, $50 \mathrm{~cm}$ environ. Avec ces morceaux, on tenta la prise de vues avec un objectif d'ouverture de F : 3,5 et un gaufrage de 225 éléments au $\mathrm{mm}^{2}$. Le gaufrage était mauvais, l'objectif trop fermé, la formule de développement pas au point, les essais échouèrent. Berthon se rendit compte qu'il fallait mettre au point un objectif de plus grande ouverture, pendant que Keller-Dorian travaillait sur l'amélioration de la machine à gaufrer. D’une intelligence supérieure, Berthon, quoi qu'aient prétendu certains de ses détracteurs, avait de très bonnes connaissances en optique. Il fit le calcul analytique d'un objectif ouvrant à 2,5 [fabriqué par Optis]. Après six mois d'études à St-Fargeau, on possédait une machine à gaufrer, des cylindres acceptables, un objectif et une formule de développement. ${ }^{10}$

Pour autant, le problème du tirage des copies était encore loin d'être résolu, selon Georges Bonnerot :

8. Anonyme, «Société du film en couleurs Keller-Dorian", rubrique "le Coin des exploitants», les Spectacles, nº 30, 25 juillet 1924, p. 14 .

9. Anonyme, "Films en couleurs Keller-Dorian ", le Journal des Finances, 23 mars 1928, p. 282.

10. Abel-Pierre Richard, "la Couleur par procédés lenticulaires", la Cinématographie française, $\mathrm{n}^{\circ}$ 647, 28 mars 1931. 
Restait alors le tirage des copies. Le problème était complexe. D’abord, l'image était inversée - au sens optique du terme - et également inversée [au développement] puisque l'épreuve était obtenue en positif directement. De plus, le support comportait un gaufrage. Se posait alors le délicat problème d'éviter, au tirage, le moirage trame sur trame ${ }^{11}$.

En 1924, il tourne quelques courtes scènes ${ }^{12}$ à Agay dans le Var, afin de procéder à des essais de tirage sur film gaufré. Mais les résultats s'avèrent inexploitables en projection.

\section{6 : Espoirs et discordes}

Durant l'année 1926, un autre événement va susciter quelques espoirs. Abel Gance qui termine son Napoléon, souhaite pouvoir tourner quelques scènes en couleurs. Les dirigeants de Keller-Dorian voient aussitôt l'intérêt pour leur société de participer à cette production prestigieuse. Une lettre en date du 26 août 1926 signée par leur administrateur, Oswald Azeray, et adressée à un destinataire inconnu, nous apprend que "Monsieur Chomon [Segundo de Chomón ${ }^{13}$ ] se rendra à Toulon pour les prises de vues concernant le film Napoléon. Il se présentera à Monsieur Abel Gance et se mettra à ses ordres ${ }^{14}$. Abel Gance tournera en effet une scène en couleurs. Une brève parue dans le Cinéopse mentionne cet épisode et Léonce-Henri Burel, opérateur pour Gance, l'a évoqué dans un entretien avec André S. Labarthe ${ }^{15}$. Il assurera beaucoup plus tard avoir également tourné quelques plans en relief, avec le procédé Cinéglyphe ${ }^{16}$, en prétendant que ce procédé restituait à la fois le relief et la couleur - ce qui reste à démontrer. L'astronome Charles Nordmann, qui s'intéresse aux recherches sur le procédé lenticulaire, est l'un des rares privilégiés ayant assisté à une projection de ces plans en couleurs. Il en rend compte dans l'Illustration:

[...] J'ai vu projeter, il y a quelques jours dans l'intimité, un fragment du Napoléon de Gance, qui par autorisation spéciale avait été pris par un appareil Keller-Dorian, en même temps qu'étaient prises les vues

11. Georges Bonnerot, "Notes sur le procédé de cinéma en couleurs de la Compagnie française Thomson-Houston ", document tapuscrit daté du 15 octobre 1976, Cinémathèque française, Fonds F. Ede, en cours de classement.

12. Ces essais, en très mauvais état, ont été conservés par les Archives Françaises du Film. La signature manuscrite de Georges Bonnerot figure sur les amorces du film, ce qui confirme ses propres notes.

13. Segundo de Chomón, est l'un des pionniers des trucages et de l'animation, rival de Méliès. Il est le co-inventeur du procédé bichrome Chomón-Zollinger (1923) proche du Kinemacolor de Urban et Smith (1911).

14. Lettre manuscrite de l'administrateur de la Société du Film en Couleurs Keller-Dorian, datée du 26 août 1926, sur papier à en-tête de la Société, Cinémathèque française, Fonds F. Ede en cours de classement.

15. Voir le Cinéopse, ${ }^{\circ}$ 85, $1^{\text {er }}$ septembre 1926 et Léonce-Henri Burel, entretien avec André S. Labarthe, dans "Abel Gance: portrait brisé» réalisé par Hubert Knapp, collection «Cinéastes de notre Temps», production ORTF, émission diffusée le 19 novembre 1964.

16. Il s'agit sans doute d'un procédé anaglyphique, (attribué à Ducos du Hauron, 1891). Il consiste à superposer sur un même support deux images stéréoscopiques aux couleurs complémentaires (cyan et rouge), qui sont visionnées avec des lunettes aux verres de même teinte. 
en noir et blanc [...] Eh bien, la comparaison est tout à fait suggestive. Sur le film Keller-Dorian, les fards violets des acteurs grimés apparaissent avec leurs vraies couleurs ${ }^{17}$ et ceux des soldats et des acteurs qui ont un joli teint naturel n'avaient pas été grimés. Mais l'un des résultats les plus curieux du nouveau procédé est qu'il supprime, en quelque sorte le cinéma en relief. Par la suite d'un mécanisme physiologique [...] le cinéma en couleurs donne la sensation du relief, sensation aussi intense que la nature même ${ }^{18}$.

Ce témoignage nuance quelque peu celui de Gance. Selon Nordmann, le film projeté n'est pas en relief et en couleurs, mais c'est cette dernière qui produit, selon lui, la sensation du relief. Ces scènes en couleurs ne furent pas utilisées au montage.

À la fin de cette même année, Robert et Sonia Delaunay réalisent un court métrage expérimental avec l'opérateur Chevereau. C'est le premier film utilisant le procédé Keller-Dorian tourné en studio et en lumière artificielle. Le fils de l'inventeur, Roland Berthon, peintre et ami des Delaunay, est sans doute à l'origine du projet. Ce film, dont il ne subsiste qu'une version incomplète, sans titre, d'environ deux minutes, est présenté lors d'une conférence de Sonia Delaunay à la Sorbonne, le 27 janvier $1927^{19}$. Il met en scène plusieurs mannequins filmés devant des fonds décorés de tissus. Cette courte bande s'achève sur un plan de Sonia Delaunay trônant au milieu de draperies aux couleurs éclatantes. Jean Mitry qui a participé au tournage rapporte cette curieuse observation :

La modification du filtrage (en faisant légèrement tourner le système optique sur son axe) modifiait graduellement les couleurs. Ayant tourné plusieurs films expérimentaux vers 1929, en compagnie de Pierre Chenal, du peintre Robert Delaunay et de l'opérateur Chevereau, je me souviens que nous étions parvenus à faire rougir une jeune fille au moyen de ce très simple procédé ${ }^{20}$.

Mais, au cours de cette même année 1926 intervient un grave désaccord entre le professeur Chrétien et la direction de Keller-Dorian qui s'obstine à vouloir lancer prématurément sur le marché des copies d'une qualité encore très discutable. Selon René Pommier, «tout ce qui avait été fait par aussi bien Chrétien que par Berthon n'était pas abouti» ${ }^{21}$. En juillet 1926, le professeur Chrétien quitte Keller-Dorian, entraînant dans son sillage Georges Bonnerot et René Pommier. Leur départ interrompt la poursuite de toute recherche sérieuse.

17. La pellicule orthochromatique étant quasiment insensible au jaune-rouge, on utilisait couramment un maquillage rose-violacé qui éclaircissait les tons chair. Ce produit de maquillage était commercialisé par Max Factor.

18. Charles Nordmann, «le Cinématographe en couleurs», l'Illustration du 10 mars 1928, no 4436, pp. 225-226.

19. Cette projection est attestée par une annonce parue dans l'hebdomadaire la Semaine à Paris, 21 janvier 1927, $\mathrm{n}^{\circ}$ 243, p. 46. Le film a été retrouvé dans une version incomplète aux Archives françaises du film; la recomposition trichrome a été effectuée par Michel Richard et François Ede en 2008.

20. Jean Mitry, op. cit., pp. 80-82.

21. Témoignage dactylographié de René Pommier daté du 31 mai 1949, Cinémathèque française, fonds F. Ede en cours de classement. 
Seul le procédé de gaufrage est assez bien maîtrisé et permet un début de commercialisation de la pellicule de prise de vues. C'est ainsi que Jacques Sauvageot, un caméraman travaillant pour les Archives de la Planète d'Albert Kahn tourne quelques courtes scènes pour tester le procédé lenticulaire ${ }^{22}$.

\section{8 : l'enlisement de la société Keller-Dorian}

À partir de 1928, la Société du Film Keller-Dorian va s'engager dans une fuite en avant. Elle parvient à intéresser Stefan Markus, un producteur suisse qui finance le premier film de fiction utilisant le procédé Keller-Dorian. Le tournage a lieu en Corse, durant l'été 1927. Le scénario est une adaptation de Mateo Falcone, une nouvelle de Prosper Mérimée. Le film est réalisé par William Delafontaine, un collaborateur d'Abel Gance. Camille Bert interprète le rôle-titre et le jeune Nicolas Roudenko, son fils. Il avait tenu le rôle de Napoléon enfant dans le film de Gance. On ne possède aucune indication sur la durée de ce film dont il ne subsiste aucune copie. Seul un entrefilet paru dans Cinémagazine rend compte d'une projection: «Pour la première fois nous avons applaudi des tableaux reproduisant exactement les couleurs de la nature ${ }^{23}$. 11 est très probable que seul l'original fut projeté à l'occasion de cette séance privée, destinée sans doute à rassurer des actionnaires devenus impatients, mais aussi à attirer de nouveaux commanditaires. «Les originaux pouvant être projetés, il était possible de créer vis-à-vis des profanes une confusion entre originaux et copies " 24 , ainsi qu'en témoignera plus tard René Pommier. Après quatre ans d'existence, la société Keller-Dorian n'a donc pas dépassé le stade expérimental, le tirage des copies reste toujours un problème et le déficit dépasse le million de francs ${ }^{25}$.

La Société pour limiter ses pertes va procéder à des cessions de brevets. La compagnie EastmanKodak qui s'intéresse au procédé dépêche son ingénieur-conseil, Raymond Edwin Crowther. Kodak acquiert le brevet pour le $16 \mathrm{~mm}$ : le Kodacolor, commercialisé dès 1928 est bien adapté au marché du film amateur. Le film inversible permet une projection directe sans tirage de copies. En 1932, sa fabrication sera définitivement arrêtée au profit du procédé Kodachrome, alors que cette même année, l'Agfacolor lenticulaire $16 \mathrm{~mm}$ est introduit sur le marché européen. Les ingénieurs d'Agfa ont perfectionné le gaufrage, et réduit la taille des dioptres à 28 micromètres (contre 40 micromètres pour le Kodacolor). La pellicule est également commercialisée pour la photographie en cartouches inversibles de $35 \mathrm{~mm}$ pour les appareils Leica et Contax.

22. Ces films tournés en 1928 et 1929 ont été restaurés avec succès pour le Musée Albert Kahn dans leurs couleurs originelles à la fin des années 1990 grâce à Alain Hairie, ingénieur de recherche au CNRS et Christian Angé, technicien du laboratoire GTC. Jacques Sauvageot sera engagé au milieu des années 1930 par la Compagnie Thomson-Houston et participera au tournage de la version en couleurs de Jour de Fête de Jacques Tati en 1949 (procédé Thomsoncolor).

23. Jan Star, Cinémagazine du 13 avril 1928, $\mathrm{n}^{\circ}$ 15, p. 68. Stefan Markus sera plus tard le directeur de production d'un second film en couleurs: Jeunes filles à marier de Jean Vallée (1935) tourné avec l'éphémère procédé FrancitaRealita.

24. Témoignage dactylographié de René Pommier, op. cit.

25. Les Spectacles du 21 juin 1929. 
Ne voulant pas admettre son échec, la Société Keller-Dorian fait courir des informations fantaisistes qui sont relayées par la presse spécialisée. C'est ainsi qu'on annonce la création d'une salle de cinéma consacrée exclusivement à la projection de films en couleurs et la construction imminente d'un vaste studio par la société des Cinéromans où seront tournés trois longs métrages en couleurs dès la première année ${ }^{26}$. On prévoit également d'utiliser le procédé pour les actualités de Pathé-Revue ainsi que pour des documentaires.

En 1928, la Société des Cinéromans produit effectivement un long métrage en couleurs, tourné en décors naturels, dans le Midi, mais le studio ne sera jamais construit. Un hebdomadaire rapporte avec enthousiasme l'achèvement de ce film de Jacques de Baroncelli :

On sait que l'excellent réalisateur s'était entouré de spécialistes du procédé Keller-Dorian grâce auxquels, la Femme du Voisin sera un film en couleurs naturelles. Les chaudes visions, les coloris merveilleux du Midi [...] ont été filmés suivant les règles de la technique nouvelle du tournage en couleurs et apparaîtront dans leur intégrale splendeur sur l'écran non plus blanc et noir, mais polychrome. ${ }^{27}$

Hélas, seuls quelques privilégiés pourront assister à une projection en couleurs des originaux lenticulaires. Dans Pour Vous, le critique Lucien Wahl donne un compte-rendu très éloigné de la description imaginaire faite par son confrère: «Il n’y a là qu'une expérience, après d'autres. On tâtonne, on cherche [...] Il n'y a encore rien de parfait [...] La projection a faibli parfois et les figures ont paru floues à plus d'un endroit " ${ }^{28}$. Dans le même hebdomadaire, quelques semaines plus tard un autre critique émet un jugement qui relève de l'exécution capitale: "Cette histoire dont nous avons parlé lorsqu'il $[s i c]$ nous fut montré en couleurs gagne généralement à être vu en noir et blanc» ${ }^{29}$. Jacques de Baroncelli tourne ensuite la Femme et le Pantin qui connaît le même sort et doit être distribué en noir et blanc.

Ces échecs successifs contribuent à jeter le discrédit sur la Société du Film en Couleurs KellerDorian qui brûle désormais ses dernières cartouches. Alors que le déficit ne cesse de se creuser, les conflits se multiplient parmi les actionnaires. On en trouve un résumé publié tardivement dans l'hebdomadaire les Spectacles ${ }^{30}$. Le journal rend compte de l'Assemblée générale extraordinaire qui a lieu en octobre 1930 dans une atmosphère houleuse, alors que la société est mise sous administration judiciaire. Les actionnaires, s'apercevant un peu tard de l'ampleur de la catastrophe, donnent libre cours à leurs reproches. Parmi les griefs exposés, on peut relever: les apports de fondateurs trop

26. Anonyme, "les Films en couleurs Keller-Dorian et la société Pathé-Consortium», rubrique «le Coin des directeurs", les Spectacles, $\mathrm{n}^{\circ}$ 237, 13 juillet 1928, p. 10.

27. Anonyme, rubrique "Petites Nouvelles», les Spectacles, no 247, 21 septembre 1928, p. 13.

28. Lucien Wahl, «la Femme du Voisin", Pour Vous, n 12, 7 février 1929, p. 4.

29. Roger Régent, «la Femme du Voisin», Pour Vous, no 23, 25 avril 1929, p. 6.

30. Anonyme, "Société Française de Cinématographie et de Photographie Films en couleurs Keller Dorian", rubrique «Informations financières», les Spectacles, no 406, 11 juin 1931, p. 4. 
largement rémunérés, une mise au point industrielle insuffisante, un procès en contrefaçon suivi de manière déplorable, une mauvaise rédaction des brevets de gravure qui a laissé le champ libre à des brevets concurrents, la construction à perte d'une usine sur un terrain dont la société n'était que locataire. Enfin sont blâmés la séparation d'avec le professeur Chrétien qui est considérée comme une faute lourde, ainsi que le départ de deux anciens collaborateurs (MM. Bonnerot et Pommier) sans qu'aient été prises les précautions nécessaires pour éviter qu'ils passent à la concurrence.

\section{L'aventure de La Société Française Cinéchromatique et la faillite de la Société Keller-Dorian}

En 1929, l'inventeur Rodolphe Berthon crée l'effet de surprise en fondant une société concurrente: la Société Française Cinéchromatique (SFC), alors que les recherches sur l'épineux problème du tirage des copies ont été abandonnées par Keller-Dorian depuis le départ du professeur Chrétien. Berthon réunit une petite équipe et s'adjoint la collaboration de René Pommier, l'un des deux ingénieurs chimistes dissidents qui avait quitté Keller-Dorian en soutien au professeur Chrétien. Berthon était-il proche de la solution? René Pommier qui a bien connu cet inventeur un peu fantasque le décrit comme "un touche-à-tout génial, mais qui ne s'intéressait pas à la poursuite d'un programme pour arriver à un résultat. Il passait d'une idée à l'autre, sans continuité dans la poursuite de la réalisation de ses idées. Il était capable de trouver une formule de révélateur et de la changer huit jours après. Mais c'est un type qui avait des idées " ${ }^{31}$. René Pommier poursuit en présentant la SFC comme une société anonyme financée par «un certain Cauderay qui, parti de pas grand-chose, était arrivé à se constituer une assez grosse fortune. [...] Il s'était associé à un fabricant de conserves de Nantes. C'était une société anonyme, mais en même temps, c'était très familial "32. Un nouveau venu, Charles Nordmann, auteur de l'article de l'Illustration cité plus haut, vient renforcer la petite équipe.

La création de la SFC va précipiter la fin de la Société du Film en Couleurs Keller-Dorian, vendue au groupe Célestin en 1930. Une nouvelle société est alors créée aux États-Unis: la Keller-Dorian Colorfilm Corporation. Elle rachète le passif de son homologue française en échange de l'exploitation des brevets. Un accord est passé avec Paramount et le laboratoire de recherche de Kodak qui va tenter de résoudre sans réel succès le problème posé par le tirage des copies. En 1936, alors que le Technicolor trichrome s'impose, Paramount renonce à utiliser le procédé lenticulaire.

Après son départ de la Société Keller-Dorian, Georges Bonnerot est engagé par le professeur Chrétien qui a mis au point un objectif anamorphique: l'Hypergonar. Le brevet sera exploité dans les années 1950 par la Fox pour le procédé Cinemascope. Georges Bonnerot est alors chargé d'effectuer les premiers tirages anamorphiques de Construire un feu, réalisé par Claude Autant-Lara. Le film est projeté dans une unique salle, le Studio de Paris, en décembre 1930 et ne rencontre pas de succès public $^{33}$. Le manque de débouchés commerciaux immédiats contraint Chrétien à se séparer de son

31. Entretien avec René Pommier réalisé par l’auteur le 11 avril 1994.

32. Ibid.

33. Voir Jean-Jacques Meusy (dir.), le Cinémascope entre art et Industrie, Paris, AFRHC, 2004, pp. 30-42. 
collaborateur. Georges Bonnerot poursuit alors sa carrière dans les laboratoires cinématographiques et, à ce titre, demeure un observateur attentif de l'évolution des techniques. Alors que la SFC survit avec peu de moyens, un nouveau concurrent reprenant le brevet de Berthon fait son apparition:

Dans les années 1930, un ingénieur de l'École Centrale de Paris, Jacques de Lassus Saint-Geniès s'intéressait à différents problèmes touchant le relief et la couleur. Il réussit à convaincre son proche parent, président du Conseil de la Compagnie Française Thomson-Houston de s'intéresser à l'étude d'un procédé qui prit le nom de Thomsoncolor. ${ }^{34}$

La compagnie franco-américaine Thomson-Houston est alors connue pour avoir installé au Havre en 1893 la première ligne de tramways électrifiée. On peine à trouver le lien qui existe entre ce domaine d'activité et l'industrie cinématographique mais, face aux ambitions de Thomson-Houston, la SFC entend défendre ses intérêts et s'opposer à ce qu'elle considère comme un pillage de ses propres brevets. Elle trouve en la personne de Charles Nordmann un ardent défenseur. Georges Bonnerot est le témoin amusé de l'incident ${ }^{35}$ :

L'Institut d'Optique était alors dirigé par le très éminent professeur Charles Fabry. Des réunions furent organisées pour la présentation des problèmes optiques d'actualité. L'une d'elles fut par exemple réservée à l'anamorphose et le professeur Chrétien présenta son Hypergonar. Le 18 décembre 1936, une séance fut consacrée au cinéma en couleurs, au cours de laquelle MM. Arnulf, Lucien et Armand Roux ${ }^{36}$ présentèrent leurs travaux, mais le clou de la séance qui avait lieu rue d'Ulm fut sans conteste un affrontement entre MM. De Lassus-St Geniès pour Thomsoncolor et Nordmann pour la Cinéchromatique, qui se lancèrent à la face les invectives les plus colorées, se reprochant mutuellement d'avoir profité des inventions de la partie adverse. Mais M. Nordmann était un redoutable jouteur et M. de Lassus prétendant une extinction de voix consécutive à un grave accident de la circulation, abandonna sans gloire le combat. Cette séance mémorable eut certainement des suites car M. Nordmann s'était fait assister d'un huissier chargé d'enregistrer les déclarations de l'adversaire. ${ }^{37}$

34. Georges Bonnerot, «Notes sur le procédé de cinéma en couleurs de la Compagnie française Thomson-Houston", op. cit.

35. Ibid.

36. Il s'agit du procédé additif Rouxcolor. La Belle Meunière (1948) de Marcel Pagnol, sera le seul long-métrage tourné avec ce procédé qui nécessitait un dispositif de projection spécial (voir infra les études consacrés au Rouxcolor).

37. Ce récit est rigoureusement exact. En 1937, Charles Nordmann a publié Une controverse sur le cinéma en couleurs, plaquette éditée à compte d'auteur qui relate les incidents qui ont émaillé la séance. La sténotypie des débats, retrace de manière piquante la joute oratoire qui opposa les adversaires. Un exemplaire de cette brochure est consultable à la Cinémathèque Française. 


\section{La vente des brevets au groupe Siemens-Halske dans les années 1930}

Alors que certains brevets commencent à tomber dans le domaine public et que les perspectives de développement de la SFC se réduisent comme une peau de chagrin, quelques sociétés étrangères vont pourtant manifester leur intérêt pour ses brevets. C'est ainsi qu'une délégation du puissant groupe Siemens-Halske de Berlin est reçue par René Pommier en 1930 :

Les Allemands sont arrivés dans les laboratoires de la Cinéchromatique, à Neuilly, où était notre laboratoire durant l'été 30. Il y avait le Dr Fischer, l'homme des brevets de Siemens: le Dr Strecker, l'homme de la mécanique qui voulait savoir comment se faisaient les cylindres [de gaufrage] et le fameux Kurt Räntsch, spécialiste de l'optique. Je leur ai fait visiter le labo. Mais [...] ils avaient déjà pratiquement acheté les brevets. J'ai été faire le premier voyage de reconnaissance à Berlin en novembre 1930. J'ai rencontré ceux qui allaient devenir mes collègues du labo 31, à Spandau ${ }^{38}$.

Les moyens mis en œuvre par Siemens sont sans comparaison avec ceux de Keller-Dorian ou de la SFC. Le laboratoire de Spandau mobilise dès 1931 une équipe d'une cinquantaine de techniciens. Le premier problème à résoudre est la fabrication d'une émulsion inversible. René Pommier, alors détaché par la SFC, étudie avec Perutz, le fabricant de pellicule, une émulsion répondant à un cahier des charges ainsi défini: le film doit être inversible, la granulation faible et la sensibilité élevée pour compenser l'absorption du filtre trichrome. Pour l'hypersensibilisation du film, l'ammoniaque utilisée jusque là ne donne pas de bons résultats, car trop volatile. René Pommier met au point avec succès une formule à base de sulfocyanure de potassium. Pour le tirage des copies, Kurt Räntsch conçoit une tireuse construite sur des données entièrement nouvelles. Les trois images de sélection imbriquées sur le film lenticulaire sont séparées puis recombinées par un système optique à trois objectifs 39 [Fig. 3].

Quatre ans de recherche auront été nécessaires pour que les techniciens de Siemens-Halske parviennent à obtenir des copies gaufrées de bonne qualité, avec un coût de fabrication assez bas. Depuis 1933, le cinéma allemand est tombé sous la coupe du Dr Goebbels, ministre de la Propagande, qui suit de près l'évolution technique et exerce des pressions pour accélérer la mise au point d'un procédé de film en couleurs capable de concurrencer le Technicolor. Non seulement le prestige du Reich est en jeu, mais le cinéma est alors considéré comme une arme puissante au service de la propagande nazie. Au début de l'année 1935, le procédé Siemens entre dans une phase industrielle. Le laboratoire Geyer de Munich est équipé en machines de développement et de tirage. Les ingénieurs de Siemens doivent surmonter un dernier problème: la perte de lumière due au filtre trichrome impose d'augmenter la puissance des lampes pour les projections sur grand écran. La société dépose alors un

\section{Ibid.}

39. DRP N $\mathrm{N}^{\circ} 752530$ : "Tireuse pour films lenticulaires», brevet allemand de Siemens-Halske et Kurt Räntsch du 10 novembre 1934, www.google.com/patents/US2070179? La date du brevet allemand figure dans le brevet américain. 


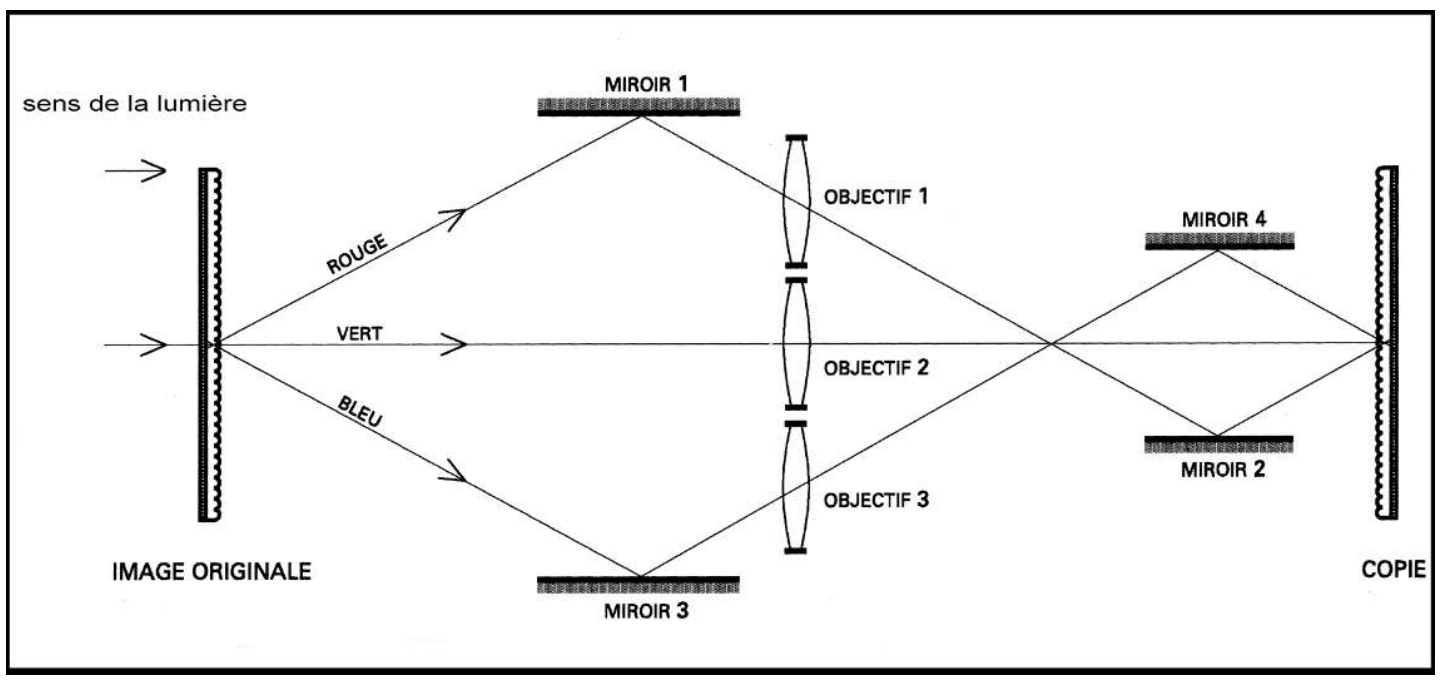

Fig. 3: Tireuse optique conçue par Kurt Räntsch. Brevet Siemens-Halske de 1934. DRP no 752530.

brevet permettant de doubler l'intensité de l'arc du projecteur et conçoit un écran spécial en aluminium offrant une meilleure réflectivité 40 .

Le 4 août 1936 a lieu la projection du premier film de fiction en couleurs tourné en SiemensOpticolor: Das Schönheitsfleckchen (la Mouche), réalisé par Rolf Hansen, d'après une nouvelle d'Alfred de Musset. La date retenue pour cette première n'est pas fortuite, elle se déroule en effet quatre jours après l'ouverture officielle des Jeux olympiques de Berlin et bénéficie d'une audience internationale. Le film reçoit un accueil enthousiaste du public, relayé par la presse berlinoise.

En septembre 1937, le film est présenté à Paris dans le cadre prestigieux de l'Exposition universelle. Deutschland, un documentaire de propagande sur Berlin réalisé par Svend Noldan est projeté en première partie et le public parisien peut découvrir l'avenue Unterdenlinden croulant sous les oriflammes d'un rouge flamboyant frappées de la croix gammée. L'organisation Kraft durch Freude [la Force par la joie] exhibe de beaux Aryens blonds à la chair rose. Nazis, mâles et femelles accompagnés de leurs petits nazillons, s'adonnent collectivement et dans un ordre impeccable à des exercices gymniques et natatoires sur les rives du Weisser See ${ }^{41}$. Le procédé rebaptisé Siemens-Berthon-Opticolor obtient le grand prix d'un jury international et est remis par le ministre du Commerce et de l'Industrie. Ce triomphe de la technique allemande sonne comme une humiliation pour l'industrie cinématographique française.

40. Edgar Gretener, "A Brief Survey of the Physics and Technology of the Berthon Siemens Color Process ", Journal of SMPE, Vol. xxviII, no 5, mai 1937, pp. 447-463.

41. Plusieurs autres courts-métrages de propagande sont présentés en Allemagne: Tag der deutschen Kunst, et Reichsparteitag Nürnberg 1937. Ce dernier film sur le congrès du parti nazi est projeté dans la salle des congrès du Deutsches Museum, qui offre 2400 places et un écran de 10 mètres de large. 
Mais alors que le triomphe de Siemens-Halske semble assuré, l'exploitation du procédé est subitement interrompue en juillet 1938. La cause de cet abandon brutal n'est pas très claire. Une version nous en est donnée par l'opérateur Hans Ertl ${ }^{42}$. En mai 1938, alors qu'il filme en Italie la visite officielle de Hitler à Mussolini, un grave incident se produit durant la prise de vues. Il ne sera découvert qu'au cours d'une projection ultérieure au laboratoire de Spandau, en présence d'un représentant du ministère de la Propagande. Un plan plonge les techniciens du laboratoire dans un état de sidération: la couleur des visages du Führer et du Duce a subitement viré au bleu de Prusse. Hertl donne une explication technique selon laquelle le filtre trichrome s'est déplacé à $180^{\circ}$ dans la monture de l'objectif, ce qui a provoqué une inversion en couleurs complémentaires ${ }^{43}$. Cet accident est à rapprocher de l'anecdote de Jean Mitry (voir supra) qui parvenait à faire rougir les jeunes filles en tournant graduellement le filtre trichrome. Mais le sens de l'humour n'a pas cours dans les officines du ministère de la Propagande. L'incident, interprété comme un acte de malveillance, aurait eu des suites immédiates et le directeur de Siemens-Halske, aussitôt convoqué au ministère, aurait été sommé de céder tous les brevets de la société à son concurrent Agfa. On ne trouve aucun élément permettant de confirmer ce témoignage peut-être apocryphe, mais il ne manque pas de saveur.

Il existe toutefois une trace écrite qui laisse supposer que le directeur de Siemens-Halske aurait subi des pressions du ministère de la Propagande afin que les recherches soient poursuivies par Agfa. En juin 1938, Paul Storch, directeur de Siemens-Halske, reconnaissant les imperfections du procédé fait acte de soumission à la direction d'Agfa et offre sa collaboration dans la poursuite des recherches ${ }^{44}$. Un mois plus tard, une réunion de crise a lieu à l'initiative de Carl Hanke, bras droit de Goebbels. Les dirigeants de Perutz, Siemens et Agfa ainsi qu'un représentant de la UFA sont convoqués. Hanke estime que les progrès du cinéma en couleurs ne sont pas suffisants et ordonne aux intéressés de faire aboutir rapidement leurs travaux de recherche afin que l'Allemagne soit enfin dotée d'un procédé de film en couleurs, capable de rivaliser avec l'industrie américaine.

En 1937, Agfa avait commercialisé un film inversible pour la photographie en couleurs. Ce procédé soustractif chromogène constituait une véritable innovation. De nombreux experts consultés alors estiment que l'Agfacolor est très supérieur au système lenticulaire et que les recherches doivent s'orienter dans cette nouvelle voie. Goebbels et Hanke, forts de ces avis, enjoignent Agfa de mettre rapidement au point un procédé négatif-positif adapté au cinéma ${ }^{45}$. Siemens-Halske se retire du jeu et, en novembre 1938, le laboratoire de Spandau cesse toute activité. Trois ans plus tard, sort le premier long métrage en Agfacolor, Frauen sind doch bessere Diplomaten (la Belle Diplomate), mis en scène par Georg Jacoby, avec en vedette Marika Rökk, alors surnommée la "Ginger Rogers nazie ».

42. Hans Ertl a travaillé notamment avec Leni Riefenstahl en 1936 sur Olympia (les Dieux du Stade).

43. Hans Ertl, Meine wilden dreißiger Jahre [Mes Folles Années trente], München, Herbig, Verlag,1982, p. 252, cité dans Dirk Mathias Alt, «les Procédés couleurs anciens et la propagande nazie (1933-1940)», mémoire de Master d'Histoire contemporaine dirigé par le Dr Füllberg-Stolberg, Université Leibniz de Hanovre, 2007, p. 62.

44. Lettre de Siemens \& Halske AG. au Dr Gajewski, directeur de IG Farbenindustrie du 27 juin 1938, Archives de l'Industrie und Filmmuseum de Wolfen, Allemagne. Fonds Agfa, cahier 11, p. 46.

45. Ibid, p. 63. Fonds Agfa, Cote A1589. 


\section{Derniers soubresauts : La compagnieThomson-Houston}

Pendant ce temps, en France, la compagnie Thomson-Houston poursuit des recherches qui s'avèrent assez peu prometteuses. Alors que la guerre approche, Georges Bonnerot va se trouver mêlé malgré lui à ces expérimentations tâtonnantes:

C'était en 1938. J'étais alors ingénieur chimiste à CTM Gennevilliers ${ }^{46}$ et le patron M. Léopold Maurice, d'ordinaire mieux inspiré, accorda l'hospitalité à Thomsoncolor [...] J'avais alors mis M. Léopold Maurice en garde devant une aventure sans aucune possibilité technique, mais je fus rudement rabroué.

En septembre 39, l'atelier Thomson qui vivotait doucement fut fermé [...] Mais en mars 40, le capitaine Jacques de Lassus attaché à la section technique de l'artillerie obtint la réouverture de l'atelier [...]. M. Léopold Maurice rappela à M. de Lassus que j'avais été avant la fermeture de l'atelier en septembre 39, l'organe de liaison entre CTM et Thomsoncolor et qu'il serait souhaitable que je reprenne cette activité bien que mobilisé. C'est alors que M. de Lassus montra qu'il était l'homme de toutes les situations. [...] Il entreprit alors des démarches auprès du Général commandant le service géographique de l'armée, dont dépendait on ne sait trop pourquoi les recherches concernant le cinéma, et obtint que je sois mis à la disposition de Thomsoncolor trois jours par semaine [...] Il faut avoir assisté à l'une de ces controverses entre un général, chef d'un service important et un capitaine besogneux fier de son nom et de la puissance qu'il représentait pour comprendre que parfois tout est possible. C'est ainsi que du 16 avril au 10 juin 40 je pris une certaine part aux travaux dirigés par M. Carlier de Calandon.

Thomsoncolor fut avant tout une affaire de famille. Ceux qui collaborèrent a' ces travaux y trouvèrent sans doute quelque profit, mais au fond travailler sans avoir la foi ou poursuivre une chimère, est-ce sérieux ${ }^{47}$ !

Après cinq années de guerre qui ont interrompu l'activité de son laboratoire, Thomson-Houston signe un accord avec le producteur Fred Orain, alors président de la Commission supérieure technique (CST). Assez naïvement, la presse de l'époque présente le procédé Thomson comme une nouveauté technique. La seule innovation notable réside dans l'abandon du tirage sur film lenticulaire:

Thomsoncolor voulant éviter les difficultés de reproduction et de projection conçut alors le processus suivant qui avait comme objectif final un film en couleurs pouvant être projeté partout.

1. Positif direct sur film gaufré

2. Extraction sur films séparés de 3 monochromes correspondant à la sélection de base.

3. Tirage sur film double couche, d'un côté la sélection du bleu, de l'autre la sélection du rouge, mordançage et coloration séparément en bleu et rouge.

4. Réémulsionnage d'une des faces, tirage de la troisième sélection, mordançage et coloration. Ouf!

La complexité du procédé, la difficulté après mordançage du film double couche de colorer séparé-

46. CTM : Cinéma Tirage Maurice, laboratoire cinématographique, fabricant de caméras (Gillon) et de matériel de laboratoire de 1919 à 1980 à Gennevilliers. Son fondateur est Léopold Gratioulet dit Maurice.

47. Georges Bonnerot, «Notes sur le procédé de cinéma en couleurs de la Compagnie française Thomson-Houston", op. cit. 
ment chaque couche, la difficulté du repérage des images à une époque où les tireuses à batteurs étaient inconnues, tout cela suffit pour rendre un tel procédé impraticable ${ }^{48}$.

La construction du laboratoire Thomsoncolor est entreprise durant l'année 1946, au 160, quai de Polangis à Nogent-sur-Marne ${ }^{49}$, sur le terrain d'une ancienne maison de passe. Fred Orain use de son autorité et de ses relations pour convaincre la profession que la France peut s'enorgueillir d'avoir trouvé enfin un procédé $100 \%$ français qu'il considère comme "supérieur au Technicolor à un coût de production nettement moindre ${ }^{50}$. Pour en faire la démonstration, il entreprend la production de Jour de Fête de Jacques Tati en 1947, alors que la construction du laboratoire Thomson n'est pas encore achevée $^{51}$. On connaît la suite: le laboratoire Thomson-Houston ne parviendra jamais à tirer un seul mètre de copie couleur et le film sera exploité uniquement dans sa version noir et blanc ${ }^{52}$. Que personne n'ait eu la lucidité de tirer les leçons des erreurs passées peut paraitre surprenant. On comprend mal en effet que ni l'État, ni aucune société privée n'aient suscité de projet industriel, alors même que les brevets Agfa étaient tombés dans le domaine public au titre des dommages de guerre. Une ordonnance du 31 juillet 1945 stipulait en effet que les brevets appartenant aux ressortissants ennemis pouvaient être cédés aux industriels français sur simple demande écrite au ministère de l'Industrie. De nombreux pays à commencer par les États-Unis profitèrent de cette aubaine et exploitèrent ces brevets avec succès. On trouve ainsi, parmi les plus connus, les procédés Eastmancolor, Ansco Color et $3 \mathrm{M}$ aux États-Unis, Gevacolor en Belgique, Ferraniacolor en Italie, Orwocolor en RDA et Sovcolor en URSS.

Le procédé Keller-Dorian, s'il reposait sur une idée élégante et prometteuse ne dépassa donc jamais véritablement le stade expérimental. Il faudra attendre près de 80 ans, pour que les travaux de Lippmann sur les microlentilles connaissent enfin un regain d'intérêt, ce qui donne la mesure de la distance qui sépare la recherche fondamentale de ses possibles applications. Cette technologie est utilisée aujourd'hui dans des domaines aussi variés que l'astronomie, l'holographie, la fibre optique, la photonique quantique et pour la vidéo, les capteurs à transfert de charges à microlentilles.

L'échec du procédé lenticulaire fut d'abord un échec industriel, dont les causes profondes peuvent être attribuées à un manque d'envergure d'un capitalisme à la française, où la spéculation et le goût de la rente l'ont emporté sur l'esprit d'entreprise. Le manque de moyens, l'amateurisme des dirigeants, les interminables conflits entre sociétés concurrentes, les batailles de brevets, et les procès qui en résultèrent furent à l'origine de ce naufrage.

\section{À la mémoire de René Pommier et Georges Bonnerot. Remerciements à Florence Paulin et Lenny Borger.}

48. Ibid.

49. Un plan et un permis de construire sont déposés le 14 mai1946. Cinémathèque française, fonds F. Ede en cours de classement.

50. Fred Orain, entretien avec François Ede, $A ̀$ la recherche de la couleur perdue, collection "Cinéma, Cinémas", produit par Claude Ventura, Michel Boujut et Anne Andreu, émission diffusée sur Antenne 2 en février 1988.

51. Ibid.

52. Voir François Ede, Jour de Fête ou la couleur retrouvée, Paris, Cahiers du Cinéma, 1995, pp. 48-49. 